\title{
Developing a Platform for Learning from Mistakes: changing the culture of patient safety amongst junior doctors
}

\author{
Sinead Millwood \\ Yeovil District Hospital NHS Foundation Trust
}

\begin{abstract}
Junior doctors commonly make mistakes which may compromise patient safety. Despite the recent push by the NHS to encourage a "no blame" culture, mistakes are still viewed as shameful, embarrassing and demoralising events. The current model for learning from mistakes means that junior doctors only learn from their own errors.
\end{abstract}

A survey was designed by the author for all the Foundation Year 1 doctors (FY1s) at Yeovil District Hospital to understand better the culture surrounding mistakes, and the types of mistakes that were being made. Using the results of the survey and the support of senior staff, a "Near misses" session has been introduced for FY1s once a month at which mistakes that have been made are discussed, with a consultant present to facilitate the proceedings. The aims of these sessions are to promote a culture of no blame, feedback information to clinical governance, and share learning experiences.

$100 \%$ of the FY1s had made a mistake that could compromise patient safety. $63 \%$ discussed their mistakes with colleagues, $44 \%$ with seniors, and only $13 \%$ with their educational supervisor. Barriers to discussing mistakes included shame, embarrassment, fear of judgement, and unapproachable seniors. 94\% thought a "Near misses" session would be useful. After the third session 100\% of the FY1s agreed that the sessions were useful; $53 \%$ had changed their practice as a result of something they learned at the sessions.

After discussing errors as a group we have worked with the clinical governance department, enacting strategies to avoid repetition of mistakes. Feedback from the junior doctors has been overwhelmingly positive and we have found these sessions to be a simple, inexpensive, and popular solution to cultural change in our organisation.

\section{Problem}

While on call during my second job as a Foundation Year 1 doctor (FY1), I made an identity error which resulted in a patient receiving an unnecessary transfusion. Despite this being a "near miss" (the patient appears not to have suffered any harm), I had never felt so awful in my life. Only 5 months earlier I had taken the Hippocratic Oath "first, do no harm" and I was already making potentially harmful mistakes. I felt ashamed, mistrustful of my ability, and alone.

I spoke to the consultant on call at the time and he reassured me that "mistakes happen", but that reflecting on the event is the best way to ensure it does not happen again. He was very kind but I continued to feel awful. I wanted to make sure nobody else made the same mistake that I did.

I began to talk to other juniors about my mistake and discovered I was not alone in the way I felt, and that most people seemed to be suffering in silence. Many felt afraid and victimised by the incident reporting system but, when asked, admitted that they had never reported an incident themselves, usually because they did not have time to fill out the cumbersome form.

The main problem I identified was a culture of fear surrounding discussing and reporting mistakes. I was concerned about the impact of this on the mental health of junior doctors and the implications for patient safety if mistakes were not being investigated.

The current system for junior doctors to deal with mistakes leaves much to be desired. We are expected to write a reflection in our eportfolio. While this can be helpful it means that only the doctor making the mistake learns from it. The reality is that, in a busy job, doctors make small mistakes on a daily basis and a reflection cannot be written for each mistake. We are encouraged to speak to our educational supervisor if we have any concerns, but this is dependent upon the degree to which the supervisor is approachable and the time available to both. The incident reporting system involves filling out a long form which may take up to $45 \mathrm{~min}$ and often does not result in any feedback.

I completed the first two of these actions and an incident report was submitted by the nurse involved in my mistake. None of these actions addressed the way I felt or educated others to learn from my mistake.

\section{Background}

It has been shown that junior doctor errors are fairly common. One publication associated with the EQUIP study, that formed the basis of the General Medical Council report "An in depth investigation into 
causes of prescribing errors by foundation trainees in relation to their medical education", found that prescribing errors are a common occurrence, affecting $7 \%$ of medication orders, $2 \%$ of patient days, and $50 \%$ of hospital admissions (1). It is somewhat concerning that with this high rate of errors, we are not discussing or processing them.

There has been a great deal of research into the negative effect that medical errors have on healthcare workers; this phenomenon has become known as the "second victim" (2). They experience many of the same emotions and feelings as the "first victims", the patient and family members (3). Initial numbness, detachment, depersonalisation, confusion, anxiety, grief, depression, withdrawal, re-experiencing of the event, shame, guilt, anger, and self doubt have all been documented as reactions to making mistakes (4).

One study of interns in France found that involvement in an adverse incident made them feel suddenly incompetent, they developed a highly negative self image and suffered negative reactions from their supervisors, feeling condemned instead of reassured. Many asserted that they did not have adequate support and would have preferred a debrief. Several interns replayed the scenario over and over in their minds and continued to think of it for more than 2 years (5). A study looking at responses to surgical complications had similar findings: "Strong emotional reactions usually faded, but memories of significant complications often lasted for years." Again institutional support was generally described as inadequate, and the participants often reported the existence of strong institutional blame cultures (6).

Wu, who coined the term "second victim", said that "Patient safety and physician welfare will be well served if we can be more honest about our mistakes to our patients, our colleagues, and ourselves". In his most recent work he calls for an increase in the recognition of the second victim phenomenon by individual practitioners, as they will be in a position to offer initial support to second victims. They can help by providing empathy and emotional support. He also discusses examples where hospitals have developed structures to support healthcare workers after involvement with an error (7). Most of these structures involve a specially trained response team to identify second victims and offer support and occasionally counselling to them.

In every hospital, individual departments discuss severe incidents at "mortality and morbidity" meetings; however, no such meetings are held for junior doctors, who are arguably the most vulnerable group of healthcare workers to the emotional effects of making a mistake. A study of residents in the USA identified a need for programmes to provide structured meaningful ways for juniors to discuss their errors, to help them cope, and to forestall negative emotional consequences. They identify that the ability to cope successfully with errors may be dependent on appropriate reassurance provided by colleagues and supervisors (8).

\section{Baseline measurement}

I designed a survey for the $21 \mathrm{FY} 1 \mathrm{~s}$ at Yeovil District Hospital to understand better the culture surrounding mistakes, and the types of mistakes that were being made. Seventeen responses were received.

The results show that over the 20 weeks preceding the questionnaire (first 5 months of FY1), there were approximately 736 errors, 91 near misses, and 73 adverse events, of which 67 were low harm, 3 moderate harm, and 3 significant harm (see attached questionnaire, question 5 , to see how these data were obtained). Most of the low harm adverse events were due to junior doctors not acting on blood results in a timely manner, forgetting to write up fluids in acute kidney injury, not stopping medications that they should have stopped, or not writing up drugs at the optimal time.

Every FY1 (100\%) had made a mistake that could potentially compromise patient safety; $69 \%$ had made an anticoagulation error, $69 \%$ an allergy prescribing error, $69 \%$ a different prescribing error, $25 \%$ a transfusion error, and $75 \%$ an identity error.

Factors which contributed to making mistakes were being on-call, time pressures, distraction, lack of support, and lack of knowledge. Sixty-three per cent of the FY1s discussed their mistakes with colleagues, $44 \%$ with seniors, and only $13 \%$ with their educational supervisor. Barriers to discussing mistakes included shame, embarrassment and fear of judgement; on further discussion many juniors felt their supervisors were not approachable.

Ninety-four per cent were in favour of starting a monthly "Near misses" session in which juniors could discuss mistakes, with a senior present to facilitate.

FY1s do not formally report their mistakes; instead, they discuss their mistakes with colleagues and occasionally, if a serious event has occurred, they will discuss them with a senior or their educational supervisor. This project has identified a culture of fear surrounding making mistakes. If we do not report or discuss mistakes then how can we learn from them and act to prevent their occurrence in the future?

See supplementary file: ds3707.pptx - "BMJ Quality questionnaire results"

\section{Design}

I designed a 1-2 h long, monthly "Near misses" session for FY1s where we could discuss our mistakes openly, with a senior doctor present to facilitate. I planned for 3-4 juniors per session to describe a mistake, what they learned from it, and what they want other people to take away from it; then we could discuss as a group ideas on how systems could be put in place to avoid repetition of that mistake. I booked the sessions as part of our mandatory weekly teaching. The aims of the sessions were to: promote a culture of no blame, share learning, and feed back information to the clinical governance department.

I discussed with and gained the support of my educational supervisor (who is also a Clinical Patient Safety Lead), and he offered to be the senior present at the first meeting. I also met with the Associate Director of Patient Safety and Quality in the clinical 
governance department, who supported the innovation and became my point of contact in that department.

Although $94 \%$ of the FY1s believed the sessions would be useful, I anticipated some resistance to discussing their mistakes due to their self confessed embarrassment, shame and fear of being judged.

\section{Strategy}

PDSA cycle 1: questionnaire - I first wanted to determine whether there was a problem. Was there a culture of fear surrounding discussion of mistakes? I wanted to know what kinds of mistakes we were making and the level of harm associated with them. I also wanted to gauge whether the idea of having a "Near misses" session was something the junior doctors would find useful. Seventeen of a total of $21 \mathrm{FY} 1 \mathrm{~s}$ completed the questionnaire. Ninety-four per cent thought a near misses session would be helpful. The rest of the information on prevalence and types of mistakes was fed back to them in a later session to reassure them that everybody has made similar mistakes and to provoke discussion about how to prevent them.

PDSA cycle 2: icebreaker - I anticipated that there would be some reluctance to discuss mistakes openly as the questionnaire had confirmed that shame, embarrassment, and fear of judgement were barriers to discussing mistakes. I conducted a preliminary $30 \mathrm{~min}$ session at the end of teaching in which I stood up and discussed my near miss and then asked each person in the room to talk for 2 min about a mistake they had made. I went in a clockwise direction around the room so that each person knew when it was their turn. It was harder for the first people but as more and more juniors admitted their mistakes, the atmosphere relaxed. One junior doctor became tearful when discussing her error, and the other juniors were very supportive. At the end I asked for a show of hands for who would like to start the sessions - the room was unanimously in favour.

PDSA cycle 3: session 1 - I planned for a $1 \mathrm{~h}$ session in which 3-4 FY1s could discuss a mistake. Each person would volunteer to come to the front and give an account of what happened. At this point the consultant could offer any comments or reassurance. Then I would ask them what they thought were the contributing factors as to why the error occurred. I intended to write a root cause analysis type diagram on the whiteboard. Then I would ask the group as a whole to make suggestions for recommendations we could make to clinical governance to help prevent that error happening again.

Unfortunately I only had $40 \mathrm{~min}$. The junior doctors were forthcoming and they wanted to discuss specific incidents, although some preferred to stay in their seat when giving an account. When it came to asking about contributing factors it became apparent that writing a diagram was too complicated and needed a person trained in root cause analysis to do it properly. We resorted to writing a list. Asking for suggestions for strategies to prevent errors was straightforward and again could be taken down in a list. We only had time to discuss two errors. The feedback I received was very positive. The juniors found it cathartic and reassuring, and they were happy that by discussing their error they may have prevented someone else repeating it. I decided that the next session would need to be $1 \mathrm{~h}$ minimum, should be less formal, and that a root cause analysis was not necessary; the focus should be on lessons learned for the juniors, recommendations to clinical governance, and promoting openness and a positive culture.

PDSA cycle 4: session 2 - With the new more relaxed agenda the session ran more smoothly; four people discussed an error and we made some very good recommendations to clinical governance. We continued the sessions in this format. I wanted to reinforce the message that everybody makes mistakes so I started the session with the well known TED talk by Dr Brian Goldman, which describes the mistakes he has made and the negative culture around discussing them (9). This enthused the junior doctors and the session was very rewarding.

PDSA cycle 5: presentation at Big Gov - I wanted the wider clinical staff to know about the sessions so that they could understand how seriously junior doctors take the mistakes that they have made and how profoundly we are affected by them. I hoped this would help to promote a culture of no blame within the wider trust. I presented the project at Big Gov, our 3-monthly trust-wide clinical governance meeting. As previously agreed, on behalf of the junior doctors I asked the staff to make our mistakes known to us, as we cannot learn from them if we do not know we have made them. I also asked our educational supervisors to initiate a conversation at our end of placement meetings about any mistakes we have made. The presentation was very well received as I believe the issue resonated with most people in the room. I received congratulations and offers of help from senior staff who were interested in attending the sessions. I think the presentation succeeded in reminding staff how much junior doctors are affected by errors and hopefully promoted a feeling of understanding, though whether this will effect a change in the culture remains to be seen.

PDSA cycle 6: Trial of Problem Based Learning and certificate- I wanted the sessions to be led by junior doctors so we trialled a Problem Based Learning approach where one junior Chairs the session and one junior is the scribe. The scribe fills out a template to describe the mistake, the learning points and any recommendations for a system change to prevent further mistakes. This template is then signed by the consultant facilitating the session and the junior can scan it into their e-portfolio. The scribe also copies the mistake and recommendations to a separate document which can be presented to the Clinical Governance Department. At Yeovil District Hospital we have started to present this information at the Patient Safety Steering Group which meets monthly. The juniors did not want the whole template to be fed back to Clinical Governance as they thought this would make people less forthcoming and destroy the anonymity which makes discussion easier. I have attached these blank documents and an explanation of how to run a "Near Misses" session in the results section.

\section{Results}

After three sessions $100 \%$ of $\mathrm{FY} 1 \mathrm{~s}$ wanted to continue them. I think 
a real testament to our success in promoting a culture of no blame is the junior doctors' willingness for further discussion of errors with other staff and with our supervisors at our end of placement meetings.

We have successfully created an environment for shared learning. Cumulatively so far we have discussed 10 incidents in detail, and 19 out of 21 juniors have discussed at least one error briefly. After three sessions $53 \%$ had changed their practice as a result of something they learned at the sessions.

After discussing errors as a group we have made a number of recommendations to the clinical governance department regarding strategies to avoid repetition of mistakes.

It is evident from our discussions that the most common mistakes are prescribing errors, specifically anticoagulation prescribing. As a result, we have raised some of our concerns and suggestions at the Safer Medicines Steering Group. Together, we identified the need for further teaching on drug interactions. We also requested feedback from the hospital pharmacy on our common prescribing errors.

A pharmacy audit of warfarin prescribing showed that $11 \%$ of international normalised ratios (INRs) $>6$ are due to incorrect prescription. Few doctors had received any previous teaching on warfarin prescribing and overall the warfarin prescription charts were found to be unclear and misleading. We have arranged elearning on warfarin for junior doctors, in addition to recommending a redesign of the warfarin chart, which is now under way. We have also recommended the use of near patient testing for INRs.

A very common error among junior doctors is the prescription of penicillin to penicillin allergic patients. There have been 16 near misses identified by this study. We have recommended introducing red allergy bands and writing the allergy status of patients on the boards above their beds, and we have asked microbiologists to introduce an allergy check during phone calls with juniors when advising them to prescribe. We believe the best way to tackle prescription errors is to introduce e-prescribing, which will be coming to the trust in the near future.

The "Near misses" sessions have initiated a dialogue between the junior doctors and the clinical governance department, previously lacking, due to few or no junior doctors filling out incident reports. This dialogue has developed to the point where it benefits both the clinical governance department and the junior doctors, providing a forum in which issues arising from the day to day performance of the junior doctor can be discussed, and providing feedback on the efficacy of quality improvement initiatives being piloted, such as the improvement of the discharge summary template and the introduction of assistant practitioners to assist the on-call team at weekends.

See supplementary file: ds3769.doc - "How to do Near Misses Meetings"

\section{Lessons and limitations}

A common problem I have encountered is ensuring a consultant is present for each session. We have run some sessions where the consultant is only present for part of the session and one where he could not attend at all. This could be overcome by drawing from a pool of consultants who are interested in attending. Interestingly, many of the junior dcotors felt they could be more open when the consultant was not present. We have drawn up a list of approachable consultants to invite to future sessions and we are asking them to open the session by discussing a mistake that they have made.

At the beginning we tried to do a mini root cause analysis after discussing an error but this was time consuming and complicated. The sessions work best when they are informal and simple.

Attendance at the sessions has been very good; it is a requirement that we attend $70 \%$ of teaching to pass FY1, and keeping the sessions within the mandatory teaching slot has worked well for us. Inevitably some juniors will be unable or unwilling to attend every session.

One junior doctor, who did not engage well at the beginning, was subsequently involved in a moderate harm adverse event and came to appreciate the importance of the sessions more than anyone else. This junior is keen to continue the sessions next year when I have moved on.

I will be doing a session during induction of the new FY1s in August, in which I hope to recruit some new FY1s to lead the sessions. This has been a very important aspect of making the sessions a success, as they should be led by a junior doctor. That way the juniors feel they are in control of the discussion and are more likely to be open and honest. We have been asked and are about to start a parallel session for FY2s.

The main limitation is the small study group, as we are a small district general hospital with $21 \mathrm{FY} 1 \mathrm{~s}$. These sessions should be piloted in other hospitals to further test their ability to promote a culture of no blame, shared learning and improve patient safety by engaging juniors in making recommendations to clinical governance. Measuring an improvement in patient safety is a notoriously difficult task and something I have not attempted to do with this project, but it may be an area of research to consider in the future.

We have found these sessions to be a simple, inexpensive, sustainable and popular solution to cultural change in our organisation and I believe they should be part of every foundation training programme.

\section{Conclusion}

Transparency and self-improvement are qualities which should be nurtured and rewarded early on in our training, especially with the emerging emphasis on the importance of candour. These sessions serve as a platform from which we can develop these qualities. I believe this project has succeeded in effecting a cultural change 


\section{BMJ Quality Improvement Reports}

surrounding the discussion of mistakes among junior doctors, from a culture of fear to a culture of openness and "no blame". The feedback I have received from the initial sessions has been overwhelmingly positive. It was difficult at first for people to admit their mistakes in front of one another, but as the sessions have progressed people are being more and more forthcoming. It is encouraging that many junior doctors have altered their practice as a result of something they learned at these meetings. Perhaps more importantly, by discussing their mistakes on a regular basis they are becoming conscientious, self-aware and self-improving practitioners, who will improve our health service in the years to come.

\section{References}

1. Lewis PJ, Dornan T, Taylor D, et al. Prevalence, incidence and nature of prescribing errors in hospital inpatients: a systematic review. Drug Saf 2009;32:379-89.

2. Wu AW. Medical error: the second victim. The doctor who makes the mistake needs help too. BMJ 2000;320:726-7.

3. Scott SD, Hirschinger LE, Cox KR, et al. The natural history of recovery for the health care provider 'second victim' after adverse patient events. Qual Saf Health Care 2009;18:325-30.

4. Schwappach DL, Boluarte TA. The emotional impact of medical error involvement on physicians: a call for leadership and organizational accountability. Swiss Med Wkly 2009;139:9-15.

5. Venus E, Galam E, Aubert J, et al. Medical errors: impact on and management by French general practitioners in training. A study of 70 questionnaires and 10 semistructured interviews. BMJ Qual Saf 2012;21:279-86

6. Pinto A, Faiz O, Bicknell C, et al.Surgical complications and their implications for surgeons' well-being. $\mathrm{Br} \mathrm{J}$ Surg 2013;100:1748-55.

7. Wu AW, Steckelberg RC. Medical error, incident investigation and the second

victim: doing better but feeling worse? BMJ Qual Saf

2012;21:267-70.

1. Kronman AC, Paasche-Orlow M, Orlander JD. Factors associated with disclosure of medical errors by housestaff. BMJ Qual Saf 2012;21:271-8.

2. Goldman D. Doctors make mistakes. Can we talk about that? TEDxToronto 2010;19:28 · Filmed Nov 2011. https://www.ted.com/talks/brian_goldman_doctors_make_mi stakes can we talk about that

\section{Declaration of interests}

Nothing to declare

\section{Acknowledgements}

Dr Zubair Khan Consultant Gastroenterologist (Patient Safety Lead), Jo Howarth Associate Director, Quality and Patient Safety,
Daniel Gibbs Clinical Audit Facilitator 